\title{
Variación espacial y temporal de nutrientes y total de sólidos en suspensión en la cuenca de un río de alta montaña tropical
}

\author{
๑C Carlos A. Benjumea-Hoyos*, \Mayra A. Suárez-Segura, @ Silvia Lucía Villabona-González \\ Grupo de investigación Limnología y Recursos Hídricos, Universidad Católica de Oriente, Rionegro, Colombia
}

\begin{abstract}
Resumen
Los sistemas de aguas dulces son sensibles a modificaciones antrópicas. Las actividades diarias allí en donde el agua es un recurso imprescindible para la industria y el ámbito doméstico aceleran el proceso natural de eutrofización en los ecosistemas acuáticos con el consecuente deterioro de su calidad. Con la finalidad de entender los cambios en el tiempo y el espacio de las concentraciones de nutrientes (nitrógeno y fósforo) y del total de sólidos en suspensión (TSS) en el río Negro, se recolectó la información en los tramos alto, medio y bajo de la cuenca en las épocas de sequía, durante la transición de la época de lluvias a la sequía y en la época de lluvias desde el 2010 hasta el 2014. Se encontró que las quebradas La Pereira, La Cimarrona y La Marinilla, afluentes del río Negro, son focos de interés ambiental, dado que presentaron mayores concentraciones de nutrientes y de sólidos en suspensión que afectan de manera notoria las aguas del río, en especial en el tramo conocido como Puente Autopista-RN5, donde estas convergen. Además, se evidenció que los fenómenos de variabilidad climática, como el sistema de El NiñoOscilación del Sur (ENOS) en su fase invernal (La Niña), incrementan notablemente las concentraciones de sólidos en suspensión y que los periodos de sequía favorecen el aumento en las concentraciones de nutrientes. C) 2018. Acad. Colomb. Cienc. Ex. Fis. Nat.
\end{abstract}

Palabras clave: Fósforo; Nitrógeno; Eutrofización; Material suspendido; Cuenca.

Temporary and spatial variation of nutrients and total suspended solids in the basin of a high mountain tropical river

\begin{abstract}
Freshwater ecosystems are highly sensitive to anthropic disturbances and daily activities in which water is an essential resource for industrial development and for domestic use have led to speed up early natural eutrophication of aquatic ecosystems resulting in water quality deterioration. In order to understand temporal and spatial changes of nutrients concentrations (nitrogen and phosphorus) and of the total suspended solids in the Río Negro, we collected information from high, medium and low basin stretches during the dry and rainy seasons, as well as during the transition from dry to rainy periods between 2010 and 2014. Our results show that Rio Negro tributary ravines La Pereira, La Cimarrona and La Marinilla are of high environmental priority as they presented the highest concentrations of nutrients and suspended solids and this is negatively affecting the Rio Negro water quality, especially in the stretch known as Puente-Autopista RN5 where these tributaries converge. In addition, we found that climatic variability phenomena such as the ENSO system, in this case, the rainy season (La Niña) seriously impact the river increasing suspended solids concentrations while the dry season favors an increase in nutrient concentrations. (C) 2018. Acad. Colomb. Cienc. Ex. Fis. Nat.
\end{abstract}

Key words: Phosphorus; Nitrogen; Eutrophication; Suspended material; Basin.

\section{Introducción}

Los sistemas de agua dulce son sensibles a modificaciones antrópicas, pues a lo largo del tiempo se han usado para depositar los desechos de la acción humana, lo que frecuentemente conlleva la desaparición de algunas de las especies que conforman las comunidades de la hidrobiota o la reducción de su diversidad en general (Lozano, 2005). Aunque los cambios naturales en estos ecosistemas, tales como los eventos catastróficos, también pueden alterar las condiciones de equilibrio, las mayores alteraciones tienen como causa las actividades antrópicas. Las actividades diarias en los lugares donde el agua es el recurso imprescindible para el desarrollo de la industria y para las actividades domésticas, así como el constante crecimiento poblacional y los cambios en los usos del suelo, habitualmente producen variaciones en las concentraciones del material en

\footnotetext{
*Correspondencia:

Carlos A. Benjumea-Hoyos, cbenjumea@uco.edu.co

Recibido: 9 de octubre de 2018

Aceptado: 17 de diciembre de 2018

Editor: Gabriel Roldán
} 
suspensión y de los nutrientes, especialmente el nitrógeno y el fósforo. Ello acelera el proceso natural de colmatación y eutrofización de los ecosistemas acuáticos debido a las descargas de aguas residuales sin ningún tratamiento previo, hecho que es usual en las grandes ciudades (Oyarzún, et al., 1997).

Es evidente que la calidad física y química del agua está relacionada con los eventos que ocurren en la cuenca y depende en gran medida del uso del suelo, del tratamiento de los efluentes de aguas servidas y del control de la erosión, entre otros. A nivel mundial hay un buen número de estudios que analizan el comportamiento de distintas variables físicas y químicas a escala de cuenca, en especial las variaciones en los parámetros asociados con los macronutrientes (nitrógeno y fósforo) y los sólidos en suspensión (Soria, et al., 1987; Rivas, et al., 2005; Rivas, et al., 2009; Giraldo, et al., 2010; Benjumea, et al., 2014; Zhang, et al., 2017; Mamun, et al., 2018). El conocimiento de las variaciones en las concentraciones de nutrientes y de sólidos en suspensión a lo largo del tiempo y en los diversos espacios de las fuentes hídricas es de gran relevancia para el desarrollo de las políticas públicas a cargo de las autoridades competentes para la adecuada gestión y control del recurso hídrico superficial en las diferentes cuencas.

Colombia es un país reconocido por la abundancia de sus recursos hídricos y por la gran diversidad biológica que albergan (IDEAM, 2014). En la región andina se originan los principales ríos del territorio nacional, los cuales surten las zonas productivas y abastecen directa o indirectamente con sus quebradas y pequeños ríos cerca del $80 \%$ de las cabeceras municipales del país. El río Negro, cuya cuenca se ubica en la región andina, es la fuente de abastecimiento de agua para consumo del municipio de Rionegro y, además, desemboca en el sistema interconectado de embalse de El Peñol - Guatapé, cuyo propósito principal es la generación hidroeléctrica (Ramírez, 1986).

Así pues, este río es uno de los principales recolectores de agua del oriente antioqueño, por lo que ha sido objeto de numerosas intervenciones humanas desde el siglo pasado para la disposición de residuos sólidos, de vertimientos domésticos e industriales y la extracción de materiales de construcción; asimismo, su cauce ha sido rectificado en algunos tramos, lo que ha llevado a la pérdida de una gran parte de la cobertura vegetal boscosa en su ronda hídrica. La fuerte presión a la que ha sido sometida su cuenca por el apresurado crecimiento de la población y del sector productivo, que demandan considerables volúmenes de agua, ha limitado la disponibilidad y la calidad de las aguas superficiales para su aprovechamiento (Corporación Autónoma Regional de las Cuencas de los Ríos Negro y Nare Cornare, 2017).

No obstante su importancia, el río Negro se ha estudiado solo a intervalos irregulares de tiempo y de sitios de muestreo, lo que genera ruido en la interpretación de la información recolectada, aunque los aportes de contaminantes de origen doméstico y agrícola continúan registrando altos niveles (Montoya, et al., 2007). El presente estudio tuvo como finalidad evaluar las variaciones en el tiempo y el espacio de las concentraciones de nutrientes (formas de nitrógeno y fósforo) y del total de sólidos suspendidos (TSS) en los diferentes tramos de la cuenca alta, media y baja del río Negro durante el periodo comprendido entre 2010 y 2014.

\section{Materiales y métodos}

Área de estudio. La cuenca del río Negro está ubicada en el departamento de Antioquia, Colombia, tiene un área de 952 $\mathrm{km}^{2}$, atraviesa los municipios de La Unión, El Carmen de Viboral, El Santuario, Guarne, La Ceja, Rionegro y Marinilla y recorre el valle de San Nicolás. Sus principales afluentes son las quebradas La Mosca, La Marinilla, La Cimarrona, La Pereira y La Chachafruto, que se convergen en el río Negro, el cual, finalmente, surte el sistema interconectado de embalses de El Peñol-Guatapé (Figura 1).

El altiplano del oriente antioqueño presenta altitudes entre los 2.100 y los $2.600 \mathrm{msnm}$ y precipitaciones que oscilan entre los 1,800 y los $2.500 \mathrm{~mm}$, con un régimen bimodal de períodos de lluvia entre abril y junio y septiembre y noviembre. Los meses de mayor precipitación son mayo y octubre y los más secos, enero y febrero (Toro, 1979).

Entre el 2010 y el 2014 se hicieron muestreos en la época de sequía (febrero-marzo), durante la transición de los periodos de lluvias a los de sequía (junio-julio) y en la época de lluvias (noviembre-diciembre) en siete tramos ubicados en el río Negro (RN1 a RN7) y en 14 ubicados en sus afluentes (quebradas Chachafruto-CH1, La Pereira-P1, P2, P3, La Cimarrona-C1, C2, C3, La Mosca-M1, M2, M3, La Marinilla-MR1, MR2 y MR3 y Compañía Abajo S1). Dichos tramos corresponden a sitios de monitorización continua establecidos por la Cornare, entidad encargada del control y la vigilancia ambiental de la zona (Tabla 1 y Figura 1).

En cada sitio de muestreo se recolectaron muestras de agua subsuperficiales siguiendo las metodologías de la American Public Health Association - APHA (2012); se analizaron las concentraciones de nitrógeno total Kjeldahl (NTK) con el método macro-Kjeldahl, de nitrógeno amoniacal (NH3-N) mediante destilación y titulación, de nitratos (NO3-N) con el método colorimétrico para cadmio y cobre, de nitritos $(\mathrm{NO} 2-\mathrm{N})$ con el mismo método y diazotización, de fósforo total mediante la digestión de ácido sulfúrico, ácido nítrico y ácido ascórbico, del total de sólidos por gravimetría y el total de sólidos en suspensión (TSS), también por gravimetría. Las muestras se analizaron en el laboratorio del Centro de Servicios de Análisis de Aguas (CENSA) de la Cornare. Además, se tomaron datos in situ del pH y el oxígeno disuelto con una sonda Hach ${ }^{\circledR}$ HQ40d.

Para evaluar el nivel de significación de las diferencias de las formas de nutrientes y los sólidos entre los factores de año, periodo y sitio de muestreo se utilizó la prueba de Kruskal-Wallis, debido a que los datos no cumplieron los supuestos de normalidad de residuos y homogeneidad de 

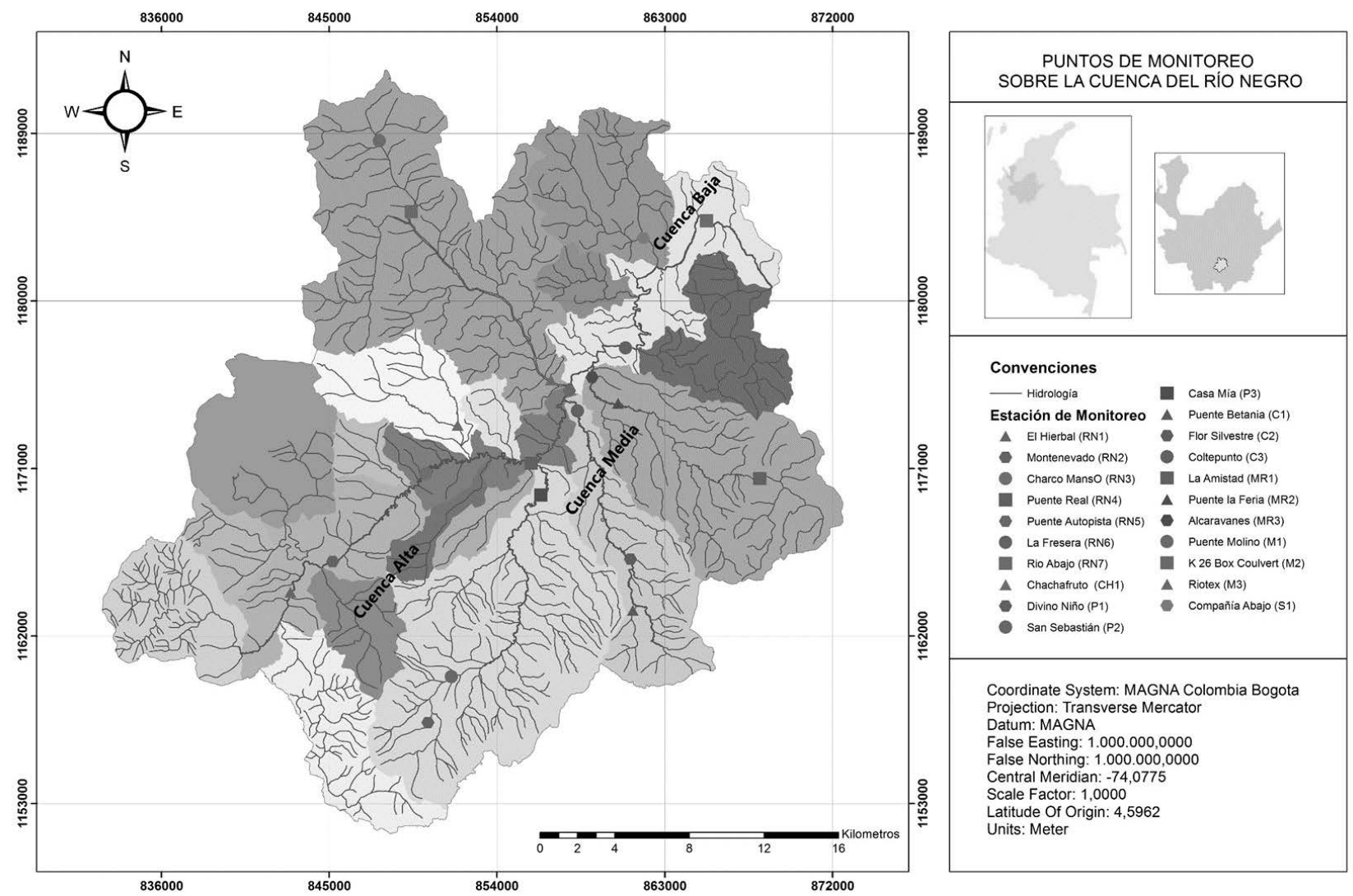

Figura 1. Área de estudio con la ubicación de los 21 sitios en la cuenca del río Negro. Fuente: elaboración propia

varianzas, incluso cuando fueron transformados mediante la raíz cuadrada y el logaritmo natural. En los casos en los que hubo diferencias significativas, se aplicó la prueba $U$ de Mann-Whitney para la comparación de las medianas. La información se procesó con el programa estadístico Rwizard, versión 2.3 (Guisande, et al., 2014).

\section{Resultados}

El $\mathrm{pH}$ varió poco y tendió a la neutralidad (mínimo $=6$ unidades; máximo $=7,65$ unidades), en tanto que el oxígeno tuvo una amplia variación, con valores entre 0,53 y 7,8 mg/l. Los tramos RN5 y RN6, ubicados en la cuenca media y baja del río Negro, en la cuenca media de La Pereira-P2 y en la cuenca baja de La Marinilla-MR3 presentaron niveles críticos $(<3,0 \mathrm{mg} / \mathrm{l})$ de oxígeno disuelto en algunos de los años $(\mathrm{P} 2=0,53 \mathrm{mg} / \mathrm{l}$ en el 2010; $2,7 \mathrm{mg} / \mathrm{l}$ en el 2011 y 1,7 $\mathrm{mg} / \mathrm{l}$ en el 2012; RN5 $=0,96 \mathrm{mg} / 1$ y RN6 $=1,02$ en el 2010; MR3 $=2,6 \mathrm{mg} / \mathrm{l}$ en el 2013, y 1,6 mg/l en el 2014).

Las concentraciones del total de sólidos en suspensión (TSS) en la cuenca tuvieron un amplio rango de variación (promedio $=52,53 \mathrm{mg} / \mathrm{l}, \mathrm{CV}=154,79 \%$ ) y oscilaron entre 2,19 y $454 \mathrm{mg} / \mathrm{l}$ en el 2010, 3 y $333 \mathrm{mg} / \mathrm{l}$ en el 2011, 3 y $172 \mathrm{mg} / \mathrm{l}$ en el 2012, 15 y 296,4 mg/l en el 2013 y 15 y $777 \mathrm{mg} / \mathrm{l}$ en el 2014. Según los resultados de las pruebas de Kruskal-Wallis y U de Mann-Whitney, los sólidos suspendidos presentaron diferencias significativas entre año y año $(\mathrm{p}<0,05)$. Específicamente el 2011 se diferenció de los demás años por presentar el valor máximo de la mediana de TSS. El 2014 se diferenció del 2012 porque en el primero de estos años se presentó una mediana mayor debido a la influencia de una gran nube de valores atípicos que incluyó las concentraciones máximas $(503 \mathrm{mg} / \mathrm{l}$ en RN5 en la transición de sequía a lluvías y $777 \mathrm{mg} / \mathrm{l}$ en MR2 en la temporada de lluvias) en tanto que en el segundo año (2012) se presentó la menor mediana de todos los años (Figura 2).

Según estas pruebas, el TSS presentó un gradiente temporal con concentraciones significativamente menores durante la sequía (febrero-marzo), seguidas del periodo de transición (junio-julio) y con las máximas concentraciones en el periodo de lluvias (noviembre-diciembre) (Figura 3). Esta variable también fue significativamente diferente en los sitios, con concentraciones máximas en el RN5 (cuenca media), el CH1 (cuenca alta), y en general en los sitios ubicados en la cuenca baja (Figura 4).

La concentración de nitritos $\left(\mathrm{NO}_{2}-\mathrm{N}\right)$ tuvo un valor medio de $0,01 \mathrm{mg} / \mathrm{l}(\mathrm{CV}=162,43 \%)$. La concentración mínima de todos los años fue de $0 \mathrm{mg} / \mathrm{l}$, en tanto que la máxima fue de $0,205 \mathrm{mg} / 1$ en el 2010 , de $0,15 \mathrm{mg} / \mathrm{l}$ en el 2011, de $0,09 \mathrm{mg} / 1$ en el 2012, de $0,03 \mathrm{mg} / \mathrm{l}$ en el 2013 y de $0,05 \mathrm{mg} / \mathrm{l}$ en el 2014. Las medianas de las concentraciones registradas en el 2012 y el 2014 fueron significativamente mayores que las de los demás años y la de la temporada de 
Tabla 1. Sitios de monitorización en la cuenca del río Negro (tomada y modificada de Cornare, 2014)

\begin{tabular}{|c|c|c|c|c|c|c|c|}
\hline \multirow[t]{2}{*}{ Cuenca } & \multirow[t]{2}{*}{ Sitio } & \multirow[t]{2}{*}{ Nombre } & \multirow[t]{2}{*}{ Ubicación } & \multicolumn{3}{|c|}{ Coordenadas } & \multirow[t]{2}{*}{ Descripción } \\
\hline & & & & $\mathbf{X}$ & $\mathbf{Y}$ & $\mathbf{Z}$ & \\
\hline \multirow[t]{4}{*}{ Alta } & RN1 & El Hierbal & \multirow[t]{3}{*}{ Río Negro } & 842916 & 1164354 & 2147 & En jurisdicción del municipio de El Retiro \\
\hline & $\mathrm{RN} 2$ & Montenevado & & 845189 & 1165991 & 2144 & $\begin{array}{l}\text { Kilómetro 5, jurisdicción del municipio } \\
\text { de El Retiro }\end{array}$ \\
\hline & RN3 & Charco Manso & & 850190 & 1170728 & 2110 & $\begin{array}{l}\text { Kilómetro 14, sector de Llanogrande } \\
\text { Jurisdicción del municipio de Rionegro }\end{array}$ \\
\hline & CH1 & FAC y Bodegas & Quebrada Chachafruto & 851929 & 1173220 & 2144 & $\begin{array}{l}\text { En jurisdicción del municipio de Rionegro. } \\
\text { Recibe las descargas de Zona Franca y } \\
\text { el aeropuerto. }\end{array}$ \\
\hline \multirow[t]{11}{*}{ Media } & RN4 & Puente Real & Río Negro & 855819 & 1171289 & 2122 & $\begin{array}{l}\text { En jurisdicción del municipio de Rionegro. } \\
\text { Recibe las descargas de la Zona Franca, } \\
\text { Hotel las Lomas y Barrio el Porvenir. }\end{array}$ \\
\hline & $\mathrm{P} 1$ & Divino Niño & $\begin{array}{l}\text { Cuenca alta quebrada } \\
\text { La Pereira }\end{array}$ & 850299 & 1157350 & 2161 & En jurisdicción del municipio de La Ceja \\
\hline & P2 & San Sebastián & $\begin{array}{l}\text { Cuenca media } \\
\text { quebrada La Pereira }\end{array}$ & 851564 & 1159824 & 2151 & $\begin{array}{l}\text { En jurisdicción del municipio de La Ceja. } \\
\text { Recibe las descargas de la PTAR de } \\
\text { ese municipio }\end{array}$ \\
\hline & $\mathrm{P} 3$ & Casa Mía & $\begin{array}{l}\text { Cuenca baja quebrada } \\
\text { La Pereira }\end{array}$ & 856234 & 1171174 & 2124 & $\begin{array}{l}\text { En jurisdicción del municipio de Rionegro. } \\
\text { Recibe algunas descargas domésticas } \\
\text { del municipio. }\end{array}$ \\
\hline & $\mathrm{C} 1$ & Puente Betania & $\begin{array}{l}\text { Cuenca alta quebrada } \\
\text { La Cimarrona }\end{array}$ & 861290 & 1163373 & 2172 & $\begin{array}{l}\text { En jurisdicción del municipio del Carmen } \\
\text { de Viboral }\end{array}$ \\
\hline & $\mathrm{C} 2$ & Flor Silvestre & $\begin{array}{l}\text { Cuenca media } \\
\text { quebrada La } \\
\text { Cimarrona }\end{array}$ & 861181 & 1166121 & 2159 & $\begin{array}{l}\text { En jurisdicción del municipio del Carmen } \\
\text { de Viboral. Recibe algunas descargas } \\
\text { domésticas y agrícolas }\end{array}$ \\
\hline & $\mathrm{C} 3$ & Coltepunto & $\begin{array}{l}\text { Cuenca baja quebrada } \\
\text { La Cimarrona }\end{array}$ & 858331 & 1174095 & 2107 & En jurisdicción del Municipio de Rionegro \\
\hline & RN5 & $\begin{array}{l}\text { Puente } \\
\text { Autopista }\end{array}$ & Río Negro & 857897 & 117517 & 2116 & $\begin{array}{l}\text { En jurisdicción de los municipios } \\
\text { de Rionegro y Marinilla. Recoge las } \\
\text { descargas de las empresas circundantes. }\end{array}$ \\
\hline & M1 & Puente Molino & $\begin{array}{l}\text { Cuenca alta quebrada } \\
\text { La Mosca }\end{array}$ & 847129 & 1188713 & 2159 & En jurisdicción del municipio de Guarne \\
\hline & M2 & $\begin{array}{l}\text { Km } 26 \text { box } \\
\text { coulvert }\end{array}$ & $\begin{array}{l}\text { Cuenca media } \\
\text { quebrada La Mosca }\end{array}$ & 849416 & 1184781 & 2160 & $\begin{array}{l}\text { En jurisdicción del municipio de Guarne. } \\
\text { Recibe algunas descargas. }\end{array}$ \\
\hline & M3 & Riotex & $\begin{array}{l}\text { Cuenca baja quebrada } \\
\text { La Mosca }\end{array}$ & 857323 & 1175448 & 2085 & $\begin{array}{l}\text { En inmediaciones de los municipios de } \\
\text { Rionegro y Marinilla. Recibe descargas de } \\
\text { las empresas circundantes. }\end{array}$ \\
\hline \multirow[t]{6}{*}{ Baja } & MR1 & La Amistad & $\begin{array}{l}\text { Cuenca alta quebrada } \\
\text { La Marinilla }\end{array}$ & 868080 & 1170499 & 2151 & En jurisdicción del municipio de Marinilla \\
\hline & MR2 & Puente La Feria & $\begin{array}{l}\text { Cuenca media } \\
\text { quebrada La Marinilla }\end{array}$ & 860494 & 1174492 & 2109 & $\begin{array}{l}\text { En jurisdicción del municipio de Marinilla. } \\
\text { Recibe descargas domésticas }\end{array}$ \\
\hline & MR3 & Alcaravanes & $\begin{array}{l}\text { Cuenca baja quebrada } \\
\text { La Marinilla }\end{array}$ & 859105 & 1175904 & 2110 & $\begin{array}{l}\text { En jurisdicción del municipio de Marinilla. } \\
\text { Recibe descargas piscícolas. }\end{array}$ \\
\hline & RN6 & La Fresera & Río Negro & 860887 & 1177457 & 2075 & $\begin{array}{l}\text { En inmediaciones de los municipios de } \\
\text { Rionegro y Marinilla }\end{array}$ \\
\hline & S1 & $\begin{array}{l}\text { Compañía } \\
\text { abajo }\end{array}$ & Quebrada El Salado & 861837 & 1183402 & 2125 & $\begin{array}{l}\text { Recibe las aguas residuales del municipio } \\
\text { de San Vicente. }\end{array}$ \\
\hline & RN7 & Río abajo & Río Negro & 865249 & 118430 & 1974 & $\begin{array}{l}\text { Ubicado en el cierre de la corriente } \\
\text { principal del río Negro, desemboca en el } \\
\text { embalse de Guatapé. }\end{array}$ \\
\hline
\end{tabular}


sequía fue mayor a las de los otros dos periodos (Figuras 2 y 3). Espacialmente, el RN5 (cuenca media), el RN7 (cuenca baja) y la cuenca baja de La Cimarrona-C3 fueron los tramos con mayor concentración de nitritos (Figura 4).

La concentración de fósforo total presentó un promedio de $0,23 \mathrm{mg} / \mathrm{l}(\mathrm{CV}=97,25 \%)$ y rangos de variación muy heterogéneos en toda la cuenca. En el 2010 las concentraciones se encontraron en un rango de 0,11 a $1,25 \mathrm{mg} / \mathrm{l}$, en el 2011 de 0,10 a $0,58 \mathrm{mg} / 1$, en el 2012 de 0,06 a 2,36 mg/l, en el 2013 de 0,05 a $0,61 \mathrm{mg} / 1$ y en el 2014 de 0,09 a 1,31 $\mathrm{mg} / \mathrm{l}$. Esta variable varió de manera significativa entre un año y otro, con concentraciones mayores en el $2010 \mathrm{y}$ el 2012 (Figura 2). Espacialmente también registró diferencias significativas al presentar concentraciones máximas en el RN5 (cuenca media) y en la cuenca media de la quebrada La Mosca-M2, donde se registró el valor máximo de todo el estudio (2,36 mg/l) durante la temporada de sequía del 2014 (Figura 4).

El nitrógeno total Kjeldahl (NTK) también presentó una gran variación, con valores entre 0,34 y 9,2 mg/l en el 2010 ; 0,29 y 7,5 mg/l en el 2011; 0,87 y $10 \mathrm{mg} / 1$ en el 2012; 1, 5,2 $\mathrm{mg} / \mathrm{l}$ en el 2013, y 1 y 10,54 mg/l en el 2014 (promedio = $1,81 \mathrm{mg} / \mathrm{l}, \mathrm{CV}=83,62 \%$ ). Los años 2010 y 2014 y los tramos medios de las quebradas La Pereira-P2, La CimarronaC2 y La Mosca-M2 se diferenciaron significativamente por presentar las mayores concentraciones de este nutriente (Figuras 2 y 4 ).

Asimismo, las concentraciones de nitrógeno amoniacal $\left(\mathrm{NH}_{3}-\mathrm{N}\right)$ tuvieron un amplio rango de variación en los años de estudio (promedio $=0,80 \mathrm{mg} / \mathrm{l}, \mathrm{CV}=120,79 \%$ ), oscilando de 0,09 a 6,2 mg/l en el 2010, de 0,2 a 2,6 mg/l en el 2011, de 0,5 a 8,6 mg/l en el 2012, de 0,4 a 2,9 mg/l en el 2013 y de 0,4 a 7,11 mg/l en el 2014. Los años 2010, 2012 y 2014 se diferenciaron significativamente de los demás porque en ellos se registraron las concentraciones máximas de amonio (Figura 2). Asimismo, el tramo RN5 ubicado en la cuenca media del río Negro, los tramos medios de las quebradas La Pereira-P2 y La Cimarrona-C2 y los tramos medio y bajo de La Mosca-M2 y M3 se diferenciaron de los demás por presentar las mayores concentraciones de esta forma de nitrógeno (Figura 4).

Los nitratos $\left(\mathrm{NO}_{3}-\mathrm{N}\right)$ tuvieron la menor fluctuación de todas las variables analizadas, con un promedio de 0,12 $\mathrm{mg} / \mathrm{l}$, un $\mathrm{CV}=72,96 \%$ y concentraciones entre 0 a 0,3 $\mathrm{mg} / \mathrm{l}$ en los años 2010, 2011 y 2012, de 0 a $0,2 \mathrm{mg} / \mathrm{l}$ en el 2013 y de 0 a $0,6 \mathrm{mg} / \mathrm{l}$ en el 2014 , es decir, no presentaron
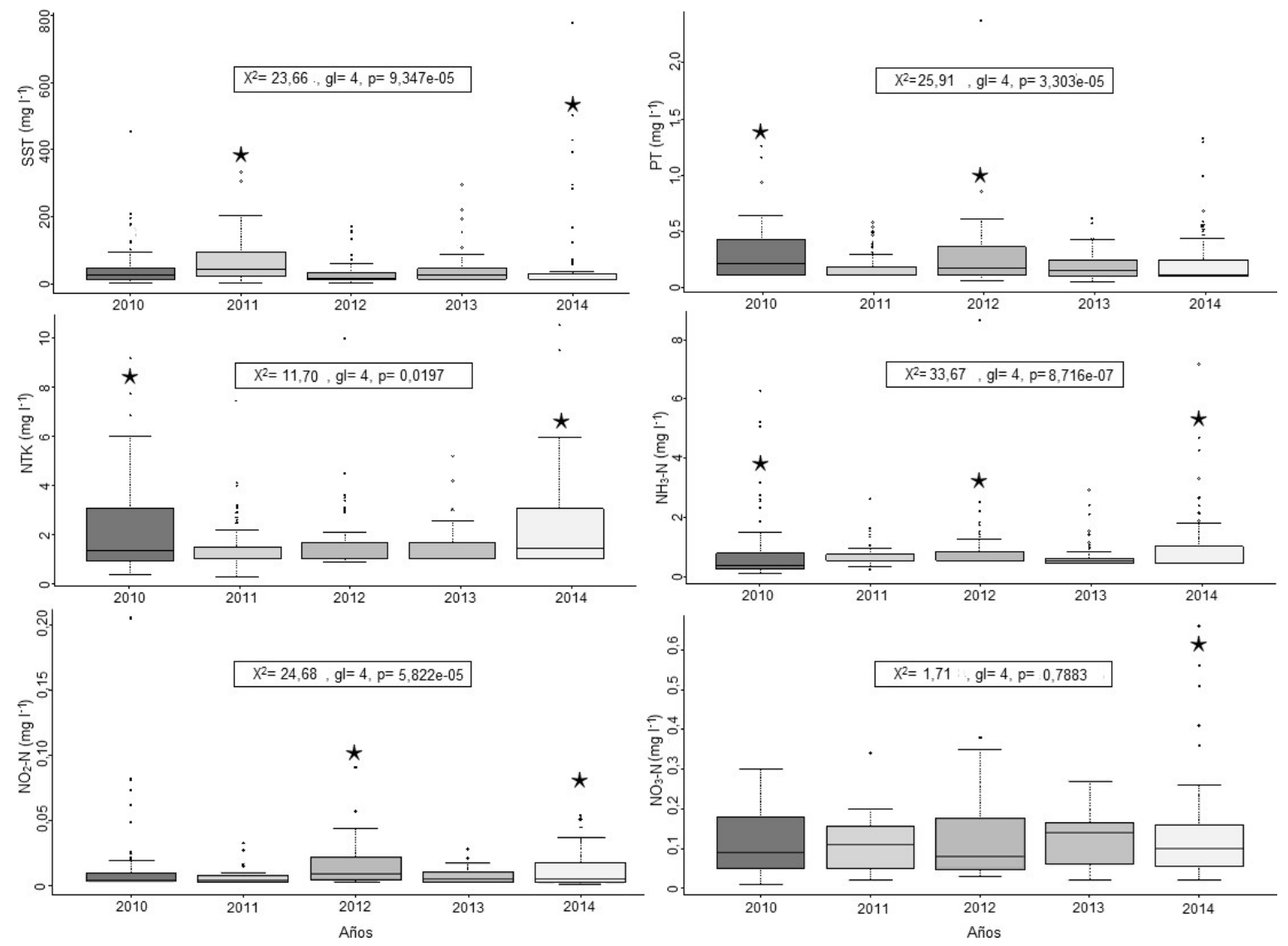

Figura 2. Diagrama de cajas y bigotes de las concentraciones de nutrientes y del total de sólidos en suspensión en los años analizados. Valores de p de Kruskal-Wallis

$\star$ Casos que presentaron diferencias significativas 

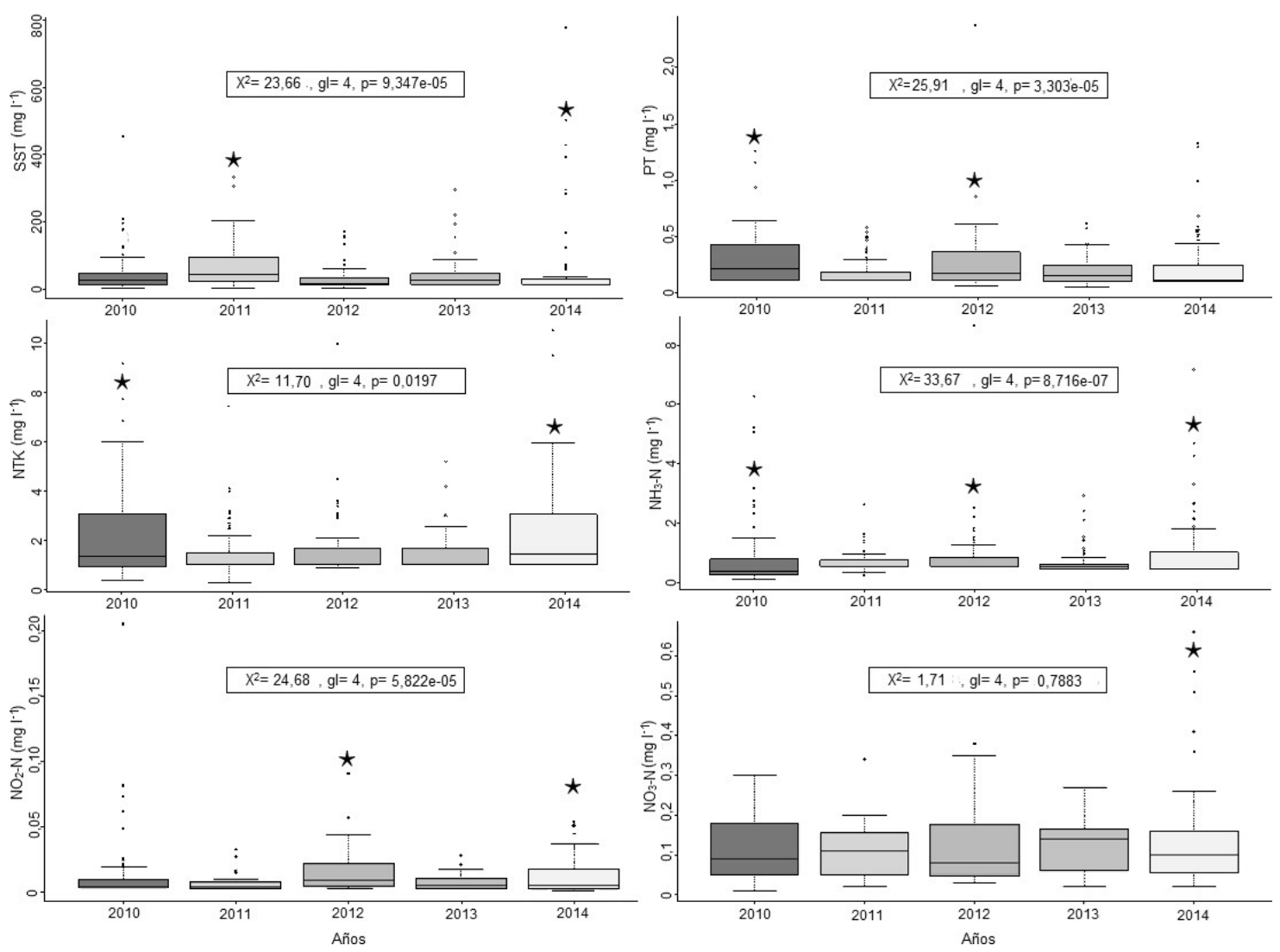

Figura 3. Diagrama de cajas y bigotes de las concentraciones de nutrientes y sólidos suspendidos totales en los periodos de muestreo. Valores de p de Kruskal-Wallis

$\star$ Casos que presentaron diferencias significativas

diferencias significativas entre los años $(\mathrm{p}>0,05)$ (Figura 2). Sin embargo, los tramos ubicados en la cuenca media y baja del río Negro, RN5, RN6 y RN7, los tres tramos de la quebrada La Pereira, P1, P2 y P2, los de La Cimarrona, C1, C2 y C3 y los de La Marinilla, MR1, MR2 y MR3, presentaron concentraciones significativamente superiores a las de los demás tramos (Figura 4).

El fósforo total, el nitrógeno total (NTK), el amonio $\left(\mathrm{NH}_{3}-\mathrm{N}\right)$ y los nitratos $\left(\mathrm{NO}_{3}-\mathrm{N}\right)$ tuvieron el mismo patrón en las temporadas de muestreo, con concentraciones significativamente mayores durante la sequía (febrero-marzo) y la transición entre la temporada de lluvias y la sequía (juniojulio) (Figura 3).

\section{Discusión}

El pH y el oxígeno disuelto (OD) son variables importantes para las actividades metabólicas de los organismos acuáticos que descomponen la materia orgánica, por lo que su variación en los sistemas acuáticos refleja la incidencia de dichos procesos y la presencia de este tipo de material. La cuenca del río Negro se encuentra en la alta montaña, por lo que el $\mathrm{pH}$ tiende a la neutralidad, algo usual en estos sistemas. Los valores de $\mathrm{pH}$ hallados para toda la cuenca presentaron similitud con lo reportado por Cadavid, et al.
(2010) para la misma zona de estudio. Roldán \& Ramírez (2008) reportaron que en aguas naturales el $\mathrm{pH}$ tiende a estar en un rango de 6,0 a 9,0 unidades. Asimismo, en ríos de alta montaña Abril, et al. (2010) reportaron valores de $\mathrm{pH}$ en ese mismo rango, y en aquellos tramos en los que se reportó tendencia hacia la acidez $(<6$ unidades de $\mathrm{pH})$ esta se asoció con la descomposición de materia orgánica. En tramos de sistemas lóticos de alta montaña en Santander, Colombia, se han obtenido valores neutros en la mayor parte de los sistemas analizados; sin embargo, algunos tramos presentaron valores ácidos $(<6$ unidades de $\mathrm{pH})$, lo cual también se atribuyó al elevado contenido de materia orgánica procedente de la vegetación circundante (Ramírez \& Plata, 2008).

La tendencia a registrar bajas concentraciones de oxígeno disuelto indica un gran consumo de este gas, posiblemente por microorganismos, ya que la oxidación de la materia orgánica es el principal factor de consumo (Roldán \& Ramírez, 2008). En muchos casos dichos valores indican hipoxia en la cuenca y reflejan, asimismo, su perturbación por la presencia de otras sustancias que ocupan el espacio de las moléculas de oxígeno. Según lo reportado por Benjumea \& Álvarez (2017), en la cuenca media del río Negro las bajas concentraciones de oxígeno se asocian principalmente 

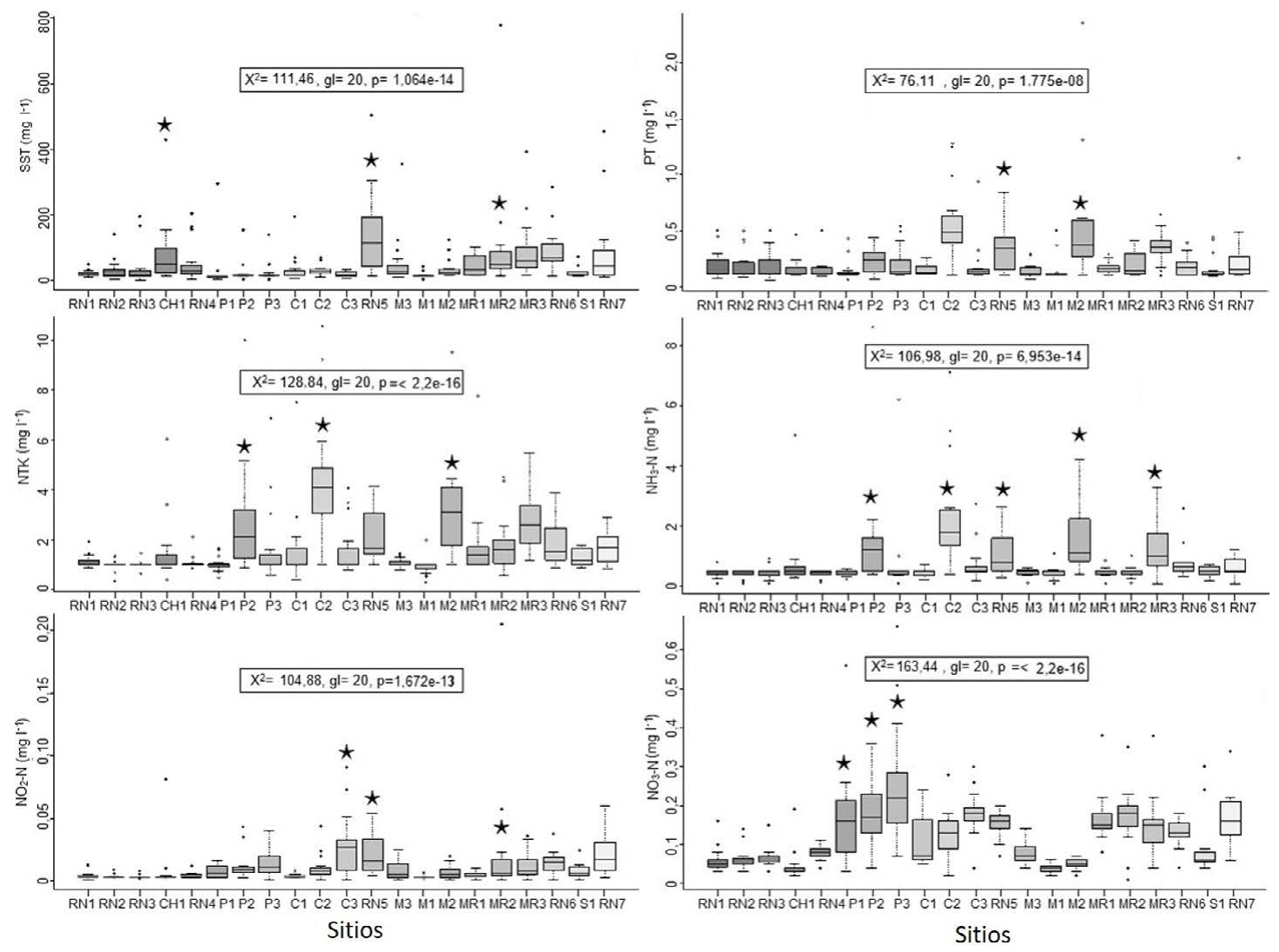

Figura 4. Diagrama de cajas y bigotes de las concentraciones de nutrientes y sólidos suspendidos totales en los sitios de muestreo del río Negro. Valores de p de Kruskal-Wallis

$\star$ Casos que presentaron diferencias significativas

con los vertimientos de origen doméstico e industrial con un escaso tratamiento y, en menor medida, con la demanda bental por los sedimentos.

Entre los tramos con niveles críticos de oxígeno disuelto está la cuenca baja de la quebrada La MarinillaMR3, cuerpo hídrico que ingresa en lo que se denomina cuenca media del río Negro. Este sitio (MR3) recibe las descargas de aguas residuales de los municipios de Marinilla y El Santuario. En este mismo sector (MR3) Cadavid, et al. (2010) reportaron un índice de calidad fisicoquímica ponderado como de calidad media, lo cual indica una afectación en el ecosistema acuático en este tramo de la cuenca que provoca el déficit de oxígeno, lo cual contribuye a la desaparición de organismos y especies presentes en ella. Además, Benjumea (2018) reportó un comportamiento similar de esta variable, registrando las concentraciones de oxígeno disuelto más bajas en la cuenca media del río Negro (Puente Autopista-RN5).

Montoya, et al. (2007) concluyeron que en el río Negro hay un elevado nivel de transporte de sólidos provenientes de la carga orgánica de las aguas residuales domesticas e industriales. Dichas concentraciones comienzan con valores bajos en la parte alta de la cuenca, pero a medida que desciende aumentan a valores medios. En el presente estudio, dicho gradiente espacial no fue muy claro en el 2011 y el 2014, cuando, en general, los sólidos en suspensión presentaron concentraciones consideradas como altas (Sierra, 2011; Ospina-Zúñiga, et al., 2015). Estos incrementos, influenciados por la variabilidad climática y las fuertes precipitaciones, son producto de la erosión del suelo (Tayupanta, 1993), siendo muy susceptibles los suelos que carecen de su cobertura vegetal, sobre todo cuando en ellos se realizan prácticas agrícolas y ganaderas inadecuadas, como ocurre en la cuenca del río Negro. En consecuencia, las altas variaciones temporales del TSS están asociadas principalmente con el ciclo hidrológico y tienden a aumentar en la época lluviosa y a disminuir en la seca. Este aumento en la carga de sólidos cobra gran relevancia en lo concerniente a la calidad del recurso hídrico y el ordenamiento de las cuencas hidrográficas, ya que tiene una relación directa con la producción de sedimentos y la contaminación de las aguas superficiales y subterráneas. En diversos estudios se ha demostrado que el deterioro ambiental de varias cuencas a nivel nacional se ha asocia con la carga de sedimentos (Restrepo, et al., 2015; Restrepo \& Escobar, 2016).

La quebrada La Marinilla, en donde se halló una concentración atípicamente alta de sólidos en suspensión en el 2014, es el tributario que aporta el mayor volumen de 
agua al río Negro, con registros de caudal en el 2010 que sobrepasaron los $25 \mathrm{~m}^{3} / \mathrm{s}$ en el sitio Alcaravanes (López, 2011). Dicha quebrada tiene una gran capacidad de arrastre de sedimentos por el caudal y por la poca presencia de vegetación en sus orillas, lo que facilita incluso más el incremento de sólidos suspendidos en la columna de agua. Además, el incremento de los sólidos en esta fuente de agua también se debe a la intervención del cauce natural a finales del 2012 para modificar el ancho del canal, ya que en épocas de invierno se presentaban graves inundaciones. A partir de ese año se han ido ejecutando proyectos en las llanuras de inundación que han generado grandes movimientos en masa, como los ocurridos en el 2014.

En cuanto a la presencia de fósforo y nitrógeno en el agua, estos macronutrientes son esenciales para el desarrollo de la actividad metabólica del sistema hídrico, por lo que las alteraciones en sus niveles de concentración hacia arriba o hacia abajo perturban al ecosistema acuático (Feria, et al., 2014). Durante el presente estudio se presentaron concentraciones considerablemente altas de fósforo, especialmente en algunos puntos de la cuenca media. Es probable que el aporte de este macronutriente provenga de la escorrentía y del lavado de las pendientes circundantes de la cuenca, lo cual propicia la erosión del suelo en donde los fertilizantes fosfatados están presentes por la actividad agrícola. En la cuenca media del río Negro Forero, et al. (2014) reportaron valores de fósforo total inclusive mayores que los registrados en este estudio, específicamente en la zona que comprende los tramos medios de las quebradas $\mathrm{La}$ Pereira-P2, La Cimarrona-C2 y La Mosca-M2, asociándolos con las actividades industriales y agropecuarias.

Las concentraciones de fósforo total registradas para toda la cuenca del río Negro durante el periodo de interés se consideran altas según varias fuentes, entre ellas la Environmental Protection Agency - EPA (2002, 2006), que consideran que concentraciones mayores a $0,04 \mathrm{mg} / 1$, como las halladas en todos los casos del presente estudio, conllevan la eutrofización de ríos y lagos. Las concentraciones aquí registradas probablemente se deben a los aportes alóctonos provenientes de las llanuras de inundación $\mathrm{y}$ de los vertimientos domésticos con escaso tratamiento, toda vez que las plantas municipales de tratamiento de aguas residuales que hacen sus vertimientos en la cuenca no cuentan con procesos terciarios, que son los indicados para la remoción de macronutrientes como el nitrógeno y el fósforo.

Estos aportes de nitrógeno y fósforo favorecen la eventual eutrofización en los sistemas leníticos, en este caso el embalse de El Peñol-Guatapé, y se relacionan directamente con lo reportado por Uribe \& Roldán (1975), Roldán, et al. (1984) y Aguirre, et al. (2007), quienes ya para la fecha de sus investigaciones clasificaban el embalse como un sistema eutrófico incipiente, y planteaban la necesidad de hacer un tratamiento adecuado de las aguas vertidas en las cuencas de influencia al embalse para la mitigación del problema.
Las concentraciones de NTK son típicas de ambientes con un grado de intervención considerable y son similares a las halladas en algunos ríos tropicales estudiados en Venezuela (Rivas, et al., 20009). Al ser este parámetro una combinación del nitrógeno orgánico en sus diversas formas con el amoniacal, su registro indica la presencia de vertimientos, posiblemente de tipo doméstico, los cuales son ricos en estos compuestos. Esto también afecta la concentración de oxígeno disuelto debido a los procesos de nitrificación que demandan los compuestos nitrogenados. Giraldo, et al. (2010) reportaron procesos similares en el río Medellín, sistema acuático urbano de montaña similar al río Negro.

Las concentraciones máximas de $\mathrm{NH}_{3}-\mathrm{N}$ registradas en el 2012 y el 2014 se consideran altas, lo cual también se registró en el río Medellín, en donde hubo condiciones críticas debido a concentraciones que han alcanzado los 8 $\mathrm{mg} / \mathrm{l}$ de $\mathrm{NH}_{3}-\mathrm{N}$ en el tramo ubicado después de la planta de tratamiento de aguas residuales de San Fernando (Giraldo, et al., 2010). Los resultados en la cuenca del río Negro reflejan que las fuentes de contaminación por nitrógeno se asocian con los vertimientos de aguas residuales y los procesos agrícolas con fertilizantes nitrogenados que llegan por escorrentía al sistema. La EPA $(2002$; 2006) considera que las concentraciones que superan los $0,76 \mathrm{mg}$ de NT/1, como las halladas en el $24 \%$ de las muestras analizadas, pueden potenciar la eutrofización en ríos y en sistemas leníticos. La Organización Mundial de la Salud (2006), por su parte, ha establecido que las concentraciones naturales de $\mathrm{NH}_{3}-\mathrm{N}$ en las aguas superficiales suelen ser menores a $0,2 \mathrm{mg} / 1$, pero cuando hay contaminación directa o difusa causada, por ejemplo, por las excretas del ganado, las concentraciones aumentan a $3 \mathrm{mg} / \mathrm{l}$. Esto se observó en el 2010 en la cuenca baja de las quebradas La Pereira-P3 y La Marinilla-MR3 y en la cuenca alta de la quebrada Chachafruto-CH1, así como en el 2012 en la cuenca media de la quebrada La Pereira-P2, y en el 2014 en la cuenca media de las quebradas La Cimarrona-C2 y La Mosca-M2, y en la cuenca baja de la quebrada La Marinilla-MR3. Por otra parte, la Agencia Europea para el Medio Ambiente - EEA, (2000, 2005) recomienda concentraciones máximas de $0,35 \mathrm{mg} \mathrm{NH}_{3}-\mathrm{N} / 1$ en los cuerpos de agua naturales, por lo que solamente el 13 $\%$ de las concentraciones halladas durante todo el periodo de estudio cumplirían con esta recomendación.

Las concentraciones del ion $\mathrm{NO}_{2}$ - son menores que las de $\mathrm{NO}_{3}$ - debido a su rápida asimilación por las bacterias responsables de la transformación a este último estado oxidativo del nitrógeno, esencial para la síntesis de proteínas, que es la forma en que las plantas y las algas lo incorporan a su sistema. Alonso (2005) reporta que valores máximos de $0,035 \mathrm{mg} / \mathrm{l}$ de $\mathrm{NO}_{2}$ - como los hallados en los muestreos de 2010, 2012 y 2014 en algunos tramos de la cuenca media y baja del río Negro (RN5, RN6 y RN7) y de las quebradas La Marinilla, Chachafruto, La Cimarrona y La Pereira, pueden ser tóxicos para los organismos. Camargo, et al. (2005) han 
señalado que una concentración máxima de $2 \mathrm{mg} / \mathrm{l}$ de $\mathrm{NO}_{3}$ tiende a perturbar las especies más sensibles del sistema. Sin embargo, en ninguno de los sitios se halló este valor en las concentraciones del ion $\mathrm{NO}_{3}$ - .

Los altos niveles de nitrógeno hallados en los afluentes La Pereira y La Cimarrona corroboran la presencia de asentamientos poblacionales en las llanuras de inundación, de prácticas de minería ilegal, de descargas no controladas y de la poca capacidad auto-depuradora de la cuenca en este tramo, a lo cual se añade, además, el déficit de oxígeno debido al consumo de los microorganismos para la oxidación de NH3-N a NO3-N.

Aunque dichos nutrientes son esenciales para los procesos enzimáticos de la producción primaria, concentraciones elevadas como las halladas conllevan la proliferación de las plantas acuáticas cuya presencia limita la disponibilidad del oxígeno, lo que proporciona ambientes anóxicos (Canet, et al., 2006), altera las condiciones de la calidad del agua y afecta la presencia y la proliferación de muchas especies de hidrobiota.

El inadecuado uso del suelo y las distintas actividades antrópicas, como la ganadería extensiva y la marcada deforestación, que se presentan en las llanuras de inundación de las quebradas La Pereira, La Cimarrona y La Marinilla (Cornare, 2006), claramente afectan la calidad del agua del tramo medio de la cuenca del río Negro, en especial la del sitio RN5. Procesos similares se evidenciaron en la cuenca del río Yeongsan (Corea del sur), donde las malas prácticas ganaderas y los aportes de las plantas de tratamiento de aguas residuales constituían la mayor fuente de aumento de estos macronutrientes (Mamun, et al., 2018).

En los demás tramos estudiados la calidad del agua fue aceptable según los resultados del estudio y concuerdan con lo reportado por Cadavid, et al. (2010) y Montoya, et al. (2011). Este comportamiento de variación espacial en el sentido del flujo de la corriente marca un gradiente característico en las concentraciones de nutrientes a medida que se avanza cuenca abajo del río Negro. Es claro, entonces, cómo un ecosistema cambia continuamente entre su cuenca alta y su desembocadura según los elementos que lo componen y sus interrelaciones (Vannote, et al., 1980). A ello se añade el proceso de asimilación, retención y transformación de los nutrientes en su recorrido aguas abajo de la cuenca (Webster \& Patten, 1979; Elwood, et al., 1981), proceso que incluye los sistemas de aprovechamiento y utilización biológica, además del transporte de nutrientes aguas abajo.

En los afluentes del río Negro se han hecho estudios que registran la mala calidad del agua con base en los índices de calidad ambiental. En un estudio previo realizado en la misma cuenca por Forero, et al. (2014), los tramos medios de las quebradas La Pereira-P2 y La Cimarrona-C2 se clasificaron como de capacidad ambiental muy baja. Especialmente el tramo P2 apareció como el punto más crítico, no solo por los vertimientos puntuales provenientes de la zona urbana del municipio de La Ceja sin ningún tratamiento previo, sino también por la presencia de suelos destinados a la actividad ganadera (Cornare, 2006).

En el estudio de Montoya, et al. (2011) en cinco sitios del río Negro, el RN5 se registró como el de peores condiciones de calidad biológica, física y química, con el agravante de que en este sector el río recibe el aporte de las quebradas La Pereira, La Cimarrona y La Mosca, todas con una calidad biológica crítica (Montoya, et al., 2007). Cornare (2017) ha señalado que la quebrada La Cimarrona pasa por toda el área urbana del municipio del Carmen de Viboral, el cual está en constante expansión poblacional, lo que incrementa la degradación de la calidad del agua. Además, sus altas pendientes propician el socavamiento del cauce, lo que incrementa los caudales (Montoya \& Montoya, 2009) y facilita el transporte del nitrógeno debido al lavado de los suelos.

El registro de mayores concentraciones de fósforo total, nitrógeno total (NTK), amonio (NH3-N) y nitratos (NO3-N) en las temporadas de sequía y transición sugiere que la presencia de materia orgánica es bastante alta y que el menor volumen del caudal en estas épocas no permite la dilución de los nutrientes. Un resultado similar se presentó en el estudio de Toja, et al. (2003) en los ríos Agrío y Guadimar en España, donde hallaron concentraciones mayores de nitrógeno y fosfatos en épocas de estiaje, atribuyendo la poca dilución de los vertidos al escaso caudal del río. Resultados similares se encontraron en el estudio previo de Montoya, et al. (2011) en el río Negro, donde los máximos valores registrados de NO3-N y fósforo total se midieron en el periodo de sequía parcial del 2007.

Al comparar los años se observó un patrón contrario, pues los valores extremos de las concentraciones de fósforo total y NO3-N en el 2010 se asociaron con un cambio sustancial en las precipitaciones por efecto del fenómeno de La Niña (García, et al., 2012; Turbay, et al., 2014), el cual produjo un aumento abrupto en aquellos caudales más proclives a producir movimientos en masa que aportan sólidos al río y propician el aumento de las concentraciones de nutrientes debido a la presencia de fertilizantes nitrogenados y excretas del ganado, los cuales son ricos en NH3-N (Ávila \& Sansores, 2003). En la cuenca del río Negro esto se intensifica debido a las altas pendientes y a los diversos usos del suelo, como el agropecuario (Cornare, 2006; 2017). En el río Fuyang, norte de China, se obtuvieron resultados parecidos, también asociados con las estaciones del clima, con mayores concentraciones de nitrógeno y fósforo en la temporada de lluvias que en la estación seca (Zhang, et al., 2017).

En conclusión, puede afirmarse que los impactos causados por los diferentes usos del suelo y la escasez de cobertura vegetal de la ronda hídrica de las quebradas La Pereira, La Cimarrona y La Mosca, ubicadas en la cuenca media, las convierten en puntos críticos de vertimiento de nutrientes $\mathrm{y}$, por lo tanto, en focos de interés ambiental en el río Negro, especialmente en el tramo Puente Autopista- 
$\mathrm{RN} 5$, y en las temporadas de sequía y transición, cuando disminuye la capacidad depuradora del río por la disminución del caudal.

En la parte baja de la cuenca, en especial el tramo Río Abajo-RN7, de gran importancia por su desembocadura en el embalse de El Peñol-Guatapé, se registraron menores concentraciones de nutrientes comparadas con los tramos restantes pese a que allí el río Negro ya ha pasado por la zona urbana del municipio de Rionegro y ha recibido los aportes de todos los tributarios que conforman la cuenca, lo cual se explicaría a su capacidad de depuración de la carga orgánica y a la reaireación que encuentra en su recorrido. Además, el tramo Río Abajo-RN7 no está sometido a la presión de los asentamientos poblacionales que generan efectos negativos en la calidad del agua.

La variación de los sólidos en suspensión se ve influenciada por fenómenos climáticos como el sistema ENOS, particularmente por la fase invernal de La Niña, que produce un incremento drástico en su concentración, principalmente en los tramos donde el cauce ha sido rectificado, dando como resultado un incremento importante en la carga de sedimentos transportados aguas abajo.

\section{Contribución de los autores}

Todos los autores participaron activamente en cada una de las fases del estudio y en la elaboración del manuscrito, así como en todo lo concerniente a la gestión, procesamiento, análisis y discusión de la información.

\section{Conflicto de interés}

Los autores declaran no tener ningún conflicto de intereses.

\section{Agradecimientos}

Los autores agradecen el apoyo del semillero de investigación Limnología y Recursos Hídricos del programa de Ingeniería Ambiental de la Universidad Católica de Oriente, y a Cornare por la colaboración y el suministro de información.

\section{Referencias}

Abril, A., Álvarez, C., Amado, A., Bonilla, C., Bueno, JC., García, J., Gutiérrez, M., Guzmán, A., Martínez, AM., Mayorga, A., Medrano, M., Moreno, N., Patiño, M., PinillaBuitrago, G., Pinto, I., Reinales, S., Rojas, A., Urrego, O. (2010). Estudio de ecosistemas terrestres y acuáticos ubicados en el Parque Nacional Natural Cueva de los Guácharos (Acevedo, Huila). Bogotá D.C., Colombia, p. 182.

Aguirre, N., Palacio, J., Ramírez, J. (2007). Características limnológicas del embalse el Peñol-Guatapé, Colombia. Revista de Ingenierías Universidad de Medellín. 6 (10): 53-66.

Alonso, A. (2005). Valoración de la degradación ambiental y efectos ecotoxicológicos sobre la comunidad de macroinvertebrados bentónicos en la cabecera del río Henares. Tesis Doctoral, Universidad de Alcalá, Alcalá de Henares (Madrid), España.

American Public Health Association - APHA. (2012). Standard methods for the examination of water and waste water. 22st ed. American Public Health Association, Washington, DC. USA: APHA, AWWA, WEF. p. 1496.
Ávila, J.P. \& Sansores, A.C. (2003). Fuentes principales de nitrógeno de nitratos en aguas subterráneas. Ingeniería Revista Académica. 7 (2): 47-54.

Benjumea, C.A. (2018). Determinación de coeficientes de degradación de materia orgánica en el río Negro. Bionatura. 3. Doi: 10.21931/RB/2018.03.01.10

Benjumea, C.A. \& Álvarez G. (2017). Demanda de oxigeno por sedimentos en diferentes tramos del río Negro, Rionegro, Antioquia, Colombia. Producción + Limpia. 12 (2): 131-146.

Benjumea, C.A., Bedoya, C., Álvarez, D. (2014). Evolución en la carga de nutrientes de ríos de montaña que fluyen a un embalse, cuenca media del río Magdalena. Revista EIA. 11 (22): 71-85.

Cadavid Gallego, J.C., Echeverri Ruiz, J.D., Gómez Gutiérrez, A.E. (2010). Modelación índices de calidad de agua (ICA) en las cuencas de la región Cornare. Gestión y Ambiente. 13 (2): 7-24.

Camargo, J.A., Alonso, A., Salamanca, A. (2005). Nitrate toxicity to aquatic animals: A review with new data for freshwater invertebrates. Chemosphere. 58: 1255-1267.

Canet, R., Ribó, M., Pomares, F., del Remedio Albiach, M. (2006). Caracterización y potenciales impactos ambientales de las deyecciones ganaderas. Conferencia sobre gestión integral de deyecciones ganaderas. Giro, gestión integral de residuos orgánicos (23-37).

Corporación Autónoma Regional de las Cuencas de los Ríos Negro y Nare - Cornare. (2006). Plan de ordenación y manejo de la cuenca La Pereira, Municipio de La Ceja Del Tambo. El Santuario.

Cornare. (2017). Cuencas. Fecha de consulta: 28 de agosto de 2017. Disponible en: http://www.cornare.gov.co/corporacion/ division-socio-ambiental/cuencas

Agencia Europea para el Medio Ambiente - EEA. (2000). Nutrients in European ecosystems. Environmental Assessment Report No 4. European Environment Agency, Copenhagen. p. 156.

Agencia Europea para el Medio Ambiente - EEA. (2005). Source apportionment of nitrogen and phosphorus inputs into the aquatic environment. Environmental Assessment Report No 7. European Environment Agency, Copenhagen. p. 52.

Elwood, J.W., Newbold J.D., Trimble, A.F., Stark, R.W. (1981). The limiting role of phosphorus in a woodland stream ecosystem: effects on P enrichment on leaf decomposition and primary producers. Ecology. 62: 146-158

Environmental Protection Agency - EPA. (2012). Nutrient Criteria Technical Guidance Manual: Rivers and Streams. Fecha de consulta: septiembre y octubre de 2017. Disponible en: http://www.epa.gov/waterscience/criteria/nutrient/ guidance/index.html

Environmental Protection Agency EPA. (2006). National recommended water quality criteria. US Environmental Protection Agency. Washington, DC. p. 23.

Feria-Díaz, J.J., Bermúdez-Roa, S., Estrada-Tordecilla, A.M. (2014). Eficiencia de la semilla Moringa oleifera como coagulante natural para la remoción de la turbidez del río Sinú. Producción + Limpia. 9 (1): 9-22.

Forero, L.C., Longo, M., Ramírez, J.J., Chalar, G. (2014). Índice de calidad ecológica con base en macroinvertebrados acuáticos para la cuenca del río Negro (ICERN-MAE), Colombia. Revista de Biología Tropical. 62 (2): 231-245. 
García, M.C., Botero, A.P., Quiroga, F.A.B., Robles, E.A. (2012). Variabilidad climática, cambio climático y el recurso hídrico en Colombia. Revista de Ingeniería. 36: 60-64.

Giraldo, L.C., Agudelo, R.A., Palacio, C.A. (2010). Spatial and temporal variation of nitrogen in the Medellin River. DYNA. 77 (163): 124-131.

Guisande, C., Heine, J., González-DaCosta, J., García-Roselló, E. (2014). RWizard Software. Vigo, España: Universidad de Vigo.

IDEAM. (2014). Estudio Nacional del Agua. Bogotá, Colombia.

López, J.D.C. (2011). Modelación hidráulica y morfodinámica de cauces sinuosos aplicación a la quebrada La Marinilla (ANT). Boletín de Ciencias de la Tierra. 30: 107-118.

Lozano, L.O. (2005). La bioindicación de la calidad del agua: importancia de los macroinvertebrados en la cuenca alta del Río Juan Amarillo, Cerros Orientales de Bogotá. Umbral Científico. 7: 5-11.

Montoya, Y., Acosta, G., Zuluaga, Z. E., García, A. (2007). Evaluación de la biodiversidad de insectos acuáticos y de calidad fisicoquímica y biológica del río Negro (Antioquia-Colombia). Revista Universidad Católica de Oriente. 23: 70-87.

Mamun, M., Lee, S. J., An, K. G. (2018). Temporal and spatial variation of nutrients, suspended solids, and chlorophyll in Yeongsan watershed. Journal of Asia-Pacific Biodiversity. 11 (2): 206-216.

Montoya, Y., Acosta, Y., Zuluaga, E. (2011). Evolución de la calidad del agua en el río Negro y sus principales tributarios empleando como indicadores los índices ICA, el BMWP/ COL y el ASPT. Caldasia. 33 (1): 193-210.

Montoya, Y. \& Montoya, B. (2009). Caracterización morfométrica de la microcuenca de la quebrada los Andes, El Carmen de Viboral, Antioquia-Colombia. Revista Ingenierías Universidad de Medellín. 8 (15): 31-38.

Organización Mundial de la Salud. (2006). Guías para la calidad del agua potable. Volumen 1. Fecha de consulta: septiembre y octubre de 2017. Disponible en: https:/www.who.int/ water_sanitation_health/dwq/gdwq3_es_fulll_lowsres.pdf

Ospina-Zúñiga, O., García-Cobas, G., Gordillo-Rivera, J., Tovar-Hernández, K. (2015). Evaluación de la turbiedad y la conductividad ocurrida en temporada seca y de lluvia en el río Combeima (Ibagué, Colombia). Ingeniería Solidaria. 12 (19): 19-36.

Oyarzún, C. E., Campos, H., Huber, A. (1997). Exportación de nutrientes en microcuencas con distinto uso del suelo en el sur de Chile (Lago Rupanco, X Región). Revista Chilena de Historia Natural. 70: 507-519.

Ramírez, C., A. M \& Plata-Díaz, Y. (2008). Diatomeas perifíticas en diferentes tramos de dos sistemas lóticos de alta montaña (Páramo de Santurbán, Norte de Santander, Colombia) y su relación con las variables ambientales. Acta Biológica Colombiana. 13: 199-215.

Ramírez, J. J. (1986). Fitoplancton de red en el embalse de El Peñol, Colombia. Actualidades Biológicas. 15 (56): 2-13.

Restrepo, J. D. \& Escobar, H. A. (2016). Sediment load trends in the Magdalena River basin (1980-2010): Anthropogenic and climate-induced causes. Geomorphology. 302: 76-91. Doi: 10.1016/j.geomorph.2016.12.013
Restrepo, J.D., Kettner, A.J., Syvitskib, J.P.M. (2015). Recent deforestation causes rapid increase in river sediment load in the Colombian Andes. Anthropocene. 10: 13-28. Doi: 10.1016/j.ancene.2015.09.001

Rivas, Z., Márquez, R., Troncone, F., Sánchez, J., Colina, M., Hernández, P. (2005). Contribución de principales ríos tributarios a la contaminación y eutrofización del Lago de Maracaibo. Ciencia. 13 (1): 68-77.

Rivas, Z., Sánchez, J., Troncone, F., Márquez, R., Ledo, H., Colina, M., Gutiérrez, E. (2009). Nitrógeno y fósforo totales de los ríos tributarios al Sistema Lago de Maracaibo, Venezuela. Interciencia. 34 (5): 308-314.

Roldán, G., Correa, M., Machado, T., Ramírez, J. J., Velásquez, L., Zuluaga, F. (1984). Estudio limnológico de la represa de El Peñol. Actualidades Biológicas. 13 (50): 94-105.

Roldán, G. \& Ramírez, J. (2008). Fundamentos de limnología neotropical $2^{\mathrm{a}}$ Edición. Medellín, Colombia: Editorial Universidad de Antioquia. p. 440.

Sierra, C. (2011). Calidad del agua, evaluación y diagnóstico. $1^{a}$ edición. Medellín, Colombia: Editorial Universidad de Medellín. p. 457.

Soria, J.M., Miracle M.R., Vicente E. (1987). Aporte de nutrientes y eutrofización de la Albufera de Valencia. Limnetica. 3 (2): 227-242.

Tayupanta, J. R. (1993). La erosión hídrica: proceso, factores y formas. INIAP Archivo Histórico. Quito. p. 18.

Toja, J., Alcalá, E., Martín, G., Solà, C., Plans, M., Burgos, M., Prat, N. (2003). Evaluación del efecto del vertido tóxico sobre la calidad del agua y los sedimentos de los ríos Agrio y Guadiamar. Sevilla, España: Ciencia y Restauración del río Guadiamar, Consejería de Medio Ambiente. p. 78-92.

Toro, G. (1979). Suelos del Departamento de Antioquia, (Tomo I). Bogotá, Colombia: Instituto Geográfico Agustín Codazzi. p. 354.

Turbay, S., Nates, B., Jaramillo, F., Vélez, J. J., Ocampo, O. L. (2014). Adaptación a la variabilidad climática entre los caficultores de las cuencas de los ríos Porce y Chinchiná, Colombia. Investigaciones Geográficas, Boletín del Instituto de Geografía. 85: 95-112.

Uribe, A., Roldán, G. (1975). Estudio comparativo de algunas características físico-químicas y biológicas del Embalse de El Peñol (Nare). Actualidades Biológicas. 4 (11): 2-12.

Vannote, R. L., Minshall, G. W., Cummings, K. W., Sedell, V., Cushing. C. E. (1980). The River Continuum Concept. Canadian Journal of Fisheries and Aquatic Sciences. 37: 130-137.

Webster, J.R. \& Patten, B.C. (1979). Effects of watershed perturbation on stream potassium and calcium dynamica. Ecological Monographs. 49: 51-72.

Zhang, W., Jin, X., Liu, D., Lang, C., Shan, B. (2017). Temporal and spatial variation of nitrogen and phosphorus and eutrophication assessment for a typical arid riverFuyang River in northern China. Journal of Environmental Sciences. 55: 41-48. 